\title{
Análisis de riesgo de un microorganismo objetable en un suplemento dietario para la liberación de lotes
}

\author{
Emilce Lorena Fernández Acosta (iD) 1 , Sonia Lorena Fretes de Aquino ${ }^{(D)}{ }^{1}$, Rocio \\ González Ruiz Díaz iD), María Gloria Domenech (iD) 1 \\ ${ }^{1}$ Universidad Nacional de Asunción, Facultad de Ciencias Químicas, Carrera de \\ Farmacia. San Lorenzo, Paraguay
}

Cómo referenciar este artículo/ How to reference this article:
Fernández E, Fretes S, González R, Domenech Ma G. Análisis de riesgo de un microorganismo objetable en un suplemento dietario para la liberación de lotes. Mem. Inst. Investig. Cienc. Salud. 2021; 19(3): $115-118$

\section{RES U M E N}

La contaminación con microorganismos objetables en los productos farmacéuticos no estériles puede tener el potencial de disminuir o inactivar la actividad farmacológica y terapéutica del principio activo y por ende representar un peligro de gran riesgo para la salud de la persona que está en tratamiento con fármacos; adicionalmente la presencia de estos microorganismos dependiendo de su capacidad para producir enfermedad en huéspedes susceptibles pueden ocasionar infecciones no esperadas 0 enfermedades que afecten al paciente o consumidor de estos medicamentos no estériles. El objetivo del trabajo fue desarrollar un análisis de riesgo microbiológico de microorganismos objetables en un suplemento dietario de una industria farmacéutica como herramienta en la gestión de seguridad. La metodología fue observacionaldescriptivo de corte transverso. En la industria farmacéutica (en el suplemento dietario) se desarrolló un análisis de riesgo estudiando todos los parámetros, teniendo como resultado, que las cápsulas blandas con aceite de Krill, no presentan microorganismo objetable del tipo patógeno, como producto de bajo riesgo, por lo que es aprobado para la liberación del lote siguiendo estos parámetros. En Paraguay no existen normativas acerca del análisis de microorganismos objetables, en comparación con otros países como Argentina, y desde hace tiempo en EE. UU y países europeos (por reportes de la FDA) por lo cual, este trabajo es relevante y de vital importancia para establecer legislaciones nacionales con miras a la fabricación de medicamentos seguros, confiables y eficaces.

Palabras claves: análisis de riesgo, microorganismos objetables, productos farmacéuticos no estériles.

\section{Risk analysis of an objectionable microorganism in a dietary supplement for batch release.}

\footnotetext{
A B S T R A C T

Contamination with objectionable microorganisms in non sterile pharmaceutical products may have the potential to decrease or inactivate the pharmacological and therapeutic activity of the active principle and therefore represent a danger of great risk to the health of the person who is being treated with drugs, additionally, the presence of these microorganisms, depending on their ability to produce disease in susceptible hosts, can cause unexpected infections or diseases that affect the patient or consumer of these non sterile drugs. The objective of the work was to develop a Fecha de recepción: Octubre 2021. Fecha de aceptación: noviembre 2021

*Autor correspondiente: Sonia Lorena Fretes de Aquino. Facultad de Ciencias Químicas-Universidad Nacional de Asunción - Km. 11 Campus Universitario. San Lorenzo, Paraguay

Teléfono: +595971976890

Email: soniafretescom@yahoo.com.ar
} 
microbiological risk analysis of objectionable microorganisms in a dietary supplement of a pharmaceutical industry as a tool in safety management. This was an observational-descriptive cross-sectional study. In the pharmaceutical industry (in the dietary supplement) a risk analysis was developed studying all the parameters, having as a result that the soft capsules with Krill oil do not present objectionable microorganisms of the pathogenic type, as a low risk product, therefore it is approved for batch release following these parameters. In Paraguay there are no regulations about the analysis of objectionable microorganisms, in comparison with other countries such as Argentina, and for a long time in the US and European countries (due to FDA reports). Therefore, this work is relevant and very vital important to establish national legislation with a view of the manufacture of safe, reliable and effective drugs.

Keywords: risk analysis, objectionable microorganisms, non sterile pharmaceuticals.

\section{INTRODUCCIÓN}

Las Buenas Prácticas de Manufactura (BPM), así como los controles de calidad a medicamentos surgieron, en respuesta a hechos graves relacionados con la falta de calidad, pureza y eficacia de alimentos y medicamentos ${ }^{(1,2)}$.

Los productos farmacéuticos no estériles permiten un máximo de carga microbiana, pero debe haber ausencia de patógenos ${ }^{(3)}$.

La contaminación con microorganismos objetables en los productos farmacéuticos no estériles puede tener el potencial de disminuir o inactivar la actividad farmacológica y terapéutica del principio activo y por ende representar un peligro de gran riesgo para la salud de la persona que está en tratamiento con fármacos ${ }^{(4)}$.

En el trabajo de investigación, se realizó el análisis de riesgo microbiológico de microorganismo objetable encontrado, aplicadas a un suplemento dietario: cápsulas blandas con aceite de Krill, tomando como base los retiros del mercado de los productos farmacéuticos no estériles, por causa mayoritaria por presencia de microorganismos objetables, con el fin de disminuir los riesgos, garantizar la inocuidad de los medicamentos y proteger la salud del paciente.

\section{MATERIALES Y MÉTODOS}

Diseño del estudio observacional-descriptivo, de corte transverso. Fue realizado en una Industria Farmacéutica (Departamento de Microbiología) de la ciudad de Asunción.

Se realizó mediante prueba bioquímica llamada API 20A-V4.0, sistema de identificación rápida para bacterias, establecidos para los controles microbiológicos según los estándares de la Farmacopea de los Estados Unidos- USP 40 - NF 35, capítulos generales $\langle 62\rangle$, $\langle 1111\rangle$, $<1113\rangle$ y $<2022\rangle$ para las diversas determinaciones realizadas ${ }^{(5)}$.

\section{RESULTADOS}

Se realizó el análisis de riesgo desarrollado en un producto farmacéutico no estéril en una industria farmacéutica: cápsulas blandas de aceite de Krill $500 \mathrm{mg}$ (suplemento dietario) en el cuál se encontró en el control higiénico del producto, la presencia de un microorganismo Clostridium sp, realizándose posteriormente un análisis de riesgo microbiológico para determinar si el microorganismo encontrado podría ser objetable o no, y por ende determinar si es factible permitir la liberación de lotes de dicho producto.

Las fases que comprende el análisis del riesgo fueron tres: evaluación del riesgo, gestión del riesgo, y comunicación del riesgo.

Acorde a los resultados obtenidos en el análisis de riesgo con todos los parámetros analizados (ver Figura 1), se determinó que solo un lote se vio afectado por la contaminación microbiana, y que de acuerdo a las pruebas bioquímicas realizadas (API $20 \mathrm{E}$ ) no corresponden a un microorganismo objetable patógeno, por lo cual, se determinó que dicho suplemento es de riesgo bajo, debido a que la incidencia de infecciones debidas al uso de productos contaminados es extremadamente baja y está asociada principalmente con la condición de salud del receptor. Las personas que se 
verían afectadas por el consumo de este producto farmacéutico no estéril serían adultos y adultos mayores, siendo estos según las categorías de RIESGO BAJO por la forma farmacéutica (del tipo sólidos orales como suplementos dietarios) y además, según pruebas microbiológicas y bioquímicas realizadas arrojando como resultado confirmatorio la presencia de Clostridium $s p$, considerado microorganismo objetable, pero de la especie no patógena, que se conocen por bibliografía, por lo tanto es apto para el uso humano y se encuentra aprobado para la liberación del lote.

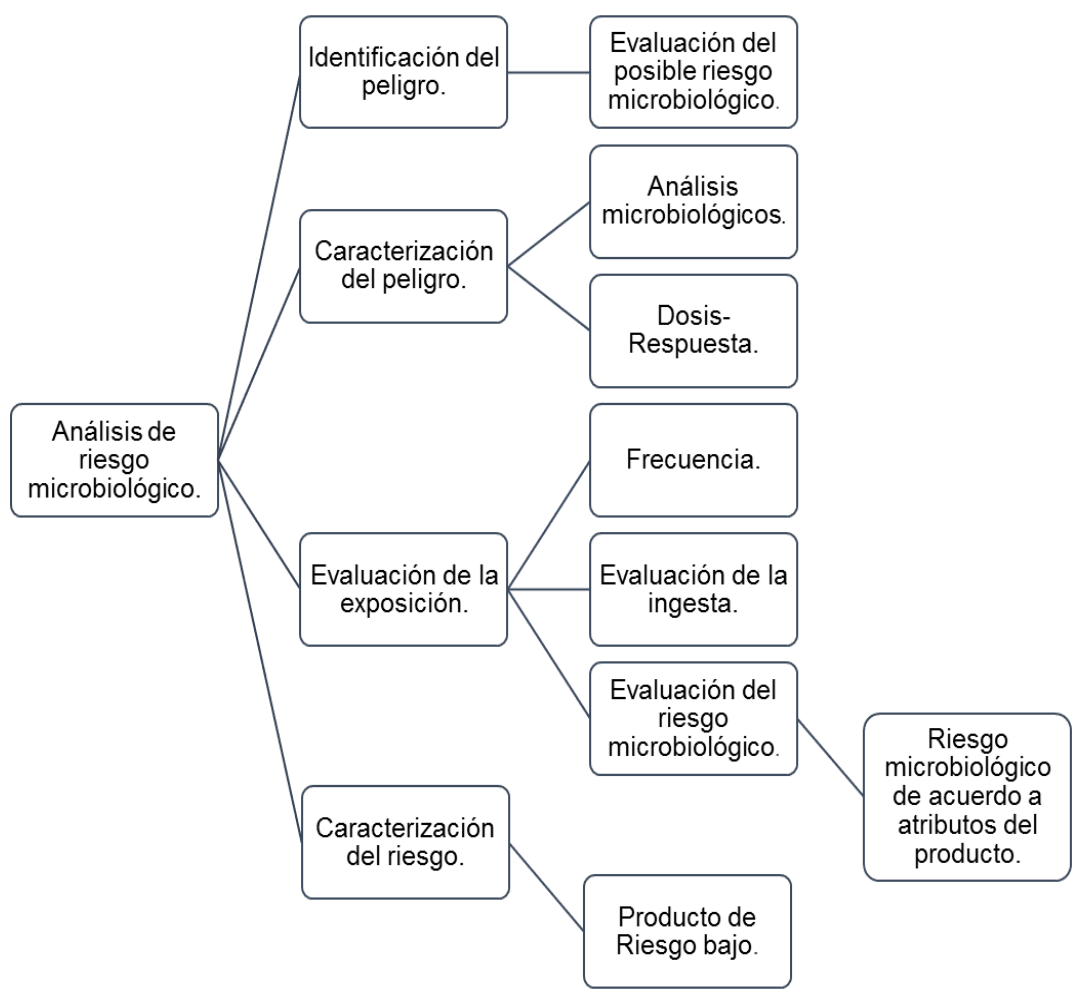

Figura 1. Análisis de riesgo de un microorganismo objetable en suplemento dietario$\mathrm{ICH}$

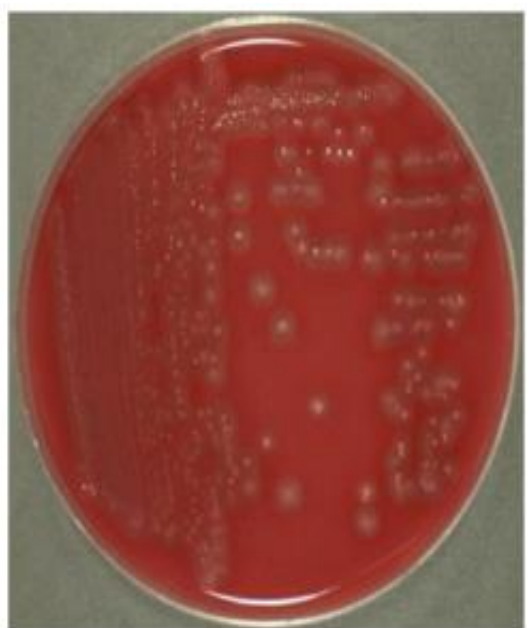

Figura 2. Cultivo en agar sangre

\section{DISCUSIÓN}

En Paraguay no existen normativas, que nos mencionen acerca de los análisis de microorganismos objetables, a diferencia con otros países de la región como Argentina, y desde hace tiempo en EE. UU y países europeos, que se reportan a través de la FDA, de ahí la importancia de la realización del análisis de riesgo a este tipo de producto, en el cual se encontró la presencia de un microorganismo objetable, el cual 
sienta las bases, para la Industria Farmacéutica para la realización de un control microbiológico más profundo, de modo a asegurar que los medicamentos fabricados sean seguros, confiables y eficaces para consumo humano. Sin embargo, existen precedentes de análisis de riesgo microbiológicos realizados en alimentos, en países de la región, así como en diferentes partes del mundo, y la aplicación de análisis de riesgos microbiológicos a productos farmacéuticos como medicamentos veterinarios en países como España ${ }^{(6)}$

En Argentina, en el año 2010 la ANMAT, se establecieron los límites microbiológicos para productos farmacéuticos no obligatoriamente estériles, y que la calidad microbiológica de los productos farmacéuticos no obligatoriamente estériles contribuye a la seguridad e inocuidad de los mismos, debido a que evidencia el cumplimiento de las Buenas Prácticas de Fabricación, debido a que los productos pueden llegar a ser vehículo de microorganismos objetables, tanto por ser patógenos, como por provocar alteración de productos, o ser indicadores de calidad higiénica deficiente ${ }^{(7-8)}$.

Se propone la realización de un plan de seguimiento a los controles microbiológicos realizados a los productos farmacéuticos no estériles, que puede consistir en procedimientos operativos estándar de pruebas microbiológicas con ligeras modificaciones, para detectar en productos a los microorganismos potencialmente objetables, y éste debe estar basado en un análisis de riesgo para determinar el nivel y probabilidad de ocurrencia de daños a la salud del paciente, de manera a seguir mejorando continuamente los controles de fabricación, la detección y la toma de decisiones para excluir los microorganismos objetables de sus productos.

\section{AGRADECIMIENTOS}

A la Industria Farmacéutica por permitir la realización de este trabajo de investigación en el departamento de Microbiología.

Conflictos de intereses: Los autores declaran no tener conflictos de intereses.

Fuentes de financiación: No tuvo financiación externa.

\section{Contribución de los autores:}

Emilce Fernández y Sonia Fretes: Concepción, diseño de la investigación, revisión de la bibliografía, recolección y análisis de datos, preparación del artículo de investigación.

Rocio González y Ma. Gloria Domenech: discusión, revisión bibliográfica y revisión del trabajo final.

\section{REFERENCIAS BIBLIOGRÁFICAS}

1. Canifarma [Internet]. México.2019:4-7. Available from:

http://codigof.mx/antecedentes-de-lasbuenas-prácticas-de-fabricación-demedicamentos.

2. Sutton $S$, Jimenez $L$. A review of reported recalls involving microbiological control 2004-2011 with emphasis on FDA considerations of "objectionable organisms." Am Pharm Rev. 2012; 15(1):1-12.

3. González LJ, Martínez FN, Rossi L, Tornese M, Troncoso A. Enfermedades transmitidas por los alimentos: Análisis del riesgo microbiológico. Rev Chil infectología. 2010; 27(6):513-24.

4. OMS. Buenas prácticas de la OMS para laboratorios de control de calidad de productos farmacéuticos. 2010;(957):148.
5. Farmacopea de los Estados Unidos, USP 38. 33a ed. Estados Unidos: United libro Press, Inc., Baltimore, MD; 2015.

6. Ratajczak M, Kubicka MM, Kamińska D, Sawicka P, Długaszewska J. Microbiological quality of non-sterile pharmaceutical products. Saudi Pharm J. 2015; 23(3): 303-7.

7. Cerra H, Aversa N, Carbone N, Carnevali S, Chiesa C, Covo M, et al. Manual de microbiología aplicada a las industrias farmacéutica, cosmética y de poductos médicos. 2013. 544 p.

8. Ogden LV, Eggett DL, Dunn ML, Jefferies LK, Taylor RH, Steele FM. Conditions associated with Clostridium sporogenes growth as a surrogate for Clostridium botulinum in nonthermally processed canned butter. J Dairy Sci [Internet]. 2013; 96(5): 2754-64. Doi: $10.3168 / j d s .2012-6209$. 